$\begin{array}{ll}\text { le portiQue } & \text { Le Portique } \\ \text { Revue de philosophie et de sciences humaines }\end{array}$

$8 \mid 2001$

Nietzsche et le divin

\title{
Nietzsche et la musique
}

\section{Pierre Montebello}

\section{OpenEdition}

\section{Journals}

\section{Édition électronique}

URL : http://journals.openedition.org/leportique/210

DOI : 10.4000/leportique.210

ISSN : $1777-5280$

\section{Éditeur}

Association "Les Amis du Portique"

Édition imprimée

Date de publication : 1 septembre 2001

ISSN : 1283-8594

\section{Référence électronique}

Pierre Montebello, « Nietzsche et la musique », Le Portique [En ligne], 8 | 2001, mis en ligne le 09 mars 2005, consulté le 12 avril 2021. URL : http://journals.openedition.org/leportique/210 ; DOI : https:// doi.org/10.4000/leportique.210

Ce document a été généré automatiquement le 12 avril 2021.

Tous droits réservés 


\title{
Nietzsche et la musique
}

\author{
Pierre Montebello
}

1 Que pensait Nietzsche de la musique de Wagner? On n'ose plus poser une telle question! À force d'entendre répéter depuis si longtemps, malgré quelques voix discordantes ici et là, que Nietzsche a dû se séparer de la musique Wagner. Et doublement encore, corps et âme. Intellectuellement parce que la musique de Wagner est ravagée par le théâtre, la grandiloquence, la démesure dramaturgique (Wagner magicien, ensorceleur, "Klingsor de tous les Klingsor", en un mot comédien). Physiologiquement parce qu'elle agit sur les nerfs comme un narcotique ou un excitant, détruit la réceptivité du corps et rend malade. Nietzsche l'a dit et répété, c'est entendu. On le répète donc après lui pour sauver Nietzsche du cas Wagner. Mais on ne s'en tient pas là! Voilà qu'on ajoute que le renoncement à Wagner a été pour Nietzsche la meilleure des choses. Se séparer de Wagner a même été un signe prophétique. Mieux, un jugement historique à mettre au crédit de Nietzsche, de quoi le distinguer et lui décerner le titre de témoin le plus lucide du déclin de la modernité. Nietzsche se serait séparé de Wagner au moment où il pressentait en lui un prélude à la modernité spectaculaire, cette sorte «de national-esthétisme» qui transforme l'art en propagande. Il aurait senti le crépusculaire danger d'une scénographie du politique qui agit sur le peuple par une amplification de tous les moyens artistiques à sa disposition.

Que pensez de cette évidence? Le bon Nietzsche face au mauvais Wagner. Le voyant historique contre l'âne politique nationaliste. Que nous avons évidemment affaire à une outrageuse amplification post-historique, que cette opposition caricaturale a ellemême un caractère spectaculaire et douteux, qu'elle est en tout cas fort éloignée de ce qui a pu se jouer entre Nietzsche et Wagner.

3 D'abord les faits : les écrits de Nietzsche montrent durant vingt ans, pour une raison ou une autre, la présence incontournable de Wagner. Aucun autre musicien, il va sans dire, et surtout aucun autre philosophe ne peut se vanter d'avoir occupé à ce point la pensée de Nietzsche. Nous le savons depuis Nietzsche, la pensée n'est pas seulement affaire de pensée. Toujours la pensée est soulevée de l'intérieur, par une expérience, une résonance, une puissance d'être. Pourquoi Nietzsche n'a-t-il jamais pu arracher sa pensée à Wagner au point de l'oublier? Parce que Wagner lui était lié essentiellement, 
profondément, philosophiquement. Ce qui veut dire que la pensée de Nietzsche a été indissociable d'une épreuve par la musique, d'une fécondation par la musique de Wagner. C'est pourquoi cette expérience est une scène originaire, en quelque sorte, à la fois philosophique et musicale.

La première rencontre avec Wagner date de novembre 1868. La première audition de Tristan de 1872. Tout de suite Nietzsche parle d'une double expérience sans aucune commune mesure. La rédaction de La Naissance de la tragédie s'en ressent: elle est profondément teintée de la pensée de Wagner sur le rapport entre civilisation et musique. Mais cette influence fécondante n'est toutefois pas une imitation servile. Déjà, en marge du texte publié, on sent que le rôle que Nietzsche assigne à la musique est différent de celui auquel pense Wagner. Les fragments posthumes de la même époque attestent en effet de l'orientation de Nietzsche vers la musique absolue alors que Wagner voudrait plutôt faire de la musique un auxiliaire du drame. Lorsque Nietzsche réalise son malentendu, en 1876, (et ce malentendu en enveloppe en réalité quantité d'autres), il prend ses distances. Mais il faut s'en faire une raison : prendre ses distances ne veut jamais dire pour Nietzsche nier ce qu'on a aimé. Si le sentiment d'une amitié incomparable ne tenait qu'aux affects, cela pourrait se produire. Mais toute amitié est communauté et monde, au delà de l'ego. Elle est donc toujours élargie par une expérience qui s'étend plus loin que le sujet, musicale ou philosophique. Une telle expérience monte comme une lumière à la surface des paroles les plus sombres de Nietzsche.

5 Il faut se rendre à l'évidence: Wagner a pu être assez profond pour insinuer de profondes pensées à Nietzsche et même, signe d'une grande amitié, de profondes critiques. Il a été en tout cas assez profond pour comprendre la profondeur de Nietzsche. Au plus lointain de l'amitié, au plus proche du silence, Nietzsche en atteste encore : «Quelqu'un a-t-il compris le moins du monde ce que je suis - m'a-t-il compris ? Un être l'a fait, et un seul : Richard Wagner, raison de plus pour douter qu'il fut vraiment Allemand... Qui, parmi mes "amis" allemands (dans ma vie, le mot "ami" s'écrit toujours avec des guillemets) aurait pu approcher de la profondeur du regard avec lequel il y a seize ans Wagner se fit mon prophète...? " ${ }^{1}$ Même si pèse sur ces propos une reconstitution historique (on se doute que le rôle de Wagner ne fut pas de se faire le prophète de Nietzsche ; Wagner avait déjà assez de mal à se faire le prophète de Wagner). Et aussi une inversion de l'histoire puisque c'est Nietzsche qui se mit à l'école de Wagner. Wagner en le défendant se défendait. Nietzsche n'oubliera toutefois jamais la lettre de défense de Wagner contre les attaques. Comme il n'oubliera pas avoir été profondément ému, animé et réconforté par Wagner à cette époque : "Pour rien au monde, je n'effacerais de ma vie les jours de Tribschen, des jours de confiance, de gaieté, de hasards sublimes - de moments profonds ». Bien après sa rupture avec Wagner, Nietzsche aura la lucidité de reconnaître cette dette même si c'est pour la magnifier et se grandir lui-même.

6 Si Nietzsche n'a su dissimuler ce qui le lie à Wagner, il n'a non plus su vraiment cacher le sentiment que sa musique lui inspire, y compris Parsifal. À la première écoute du prélude de Parsifal, il s'empresse d'écrire à Peter Gast : « Netteté de la musique comme art descriptif, qui fait penser à un bouclier d'une facture achevée et pour finir un sentiment sublime et extraordinaire, une expérience, une sensation de l'âme dans le fond même de la musique qui fait le plus grand honneur à Wagner"? Élévation sans équivalent, connaissance et pénétration sans pareil, synthèse d'états psychologiques 
d'une hauteur incomparable ${ }^{2}$ ! Difficile d'imaginer un éloge plus net. Mais on lui opposera certainement tout ce que Wagner attribue à Bizet. Sans entrer dans une fastidieuse confrontation de textes sensée établir une échelle du mérite, remarquons simplement que trois mois plus tard Nietzsche les met tout simplement sur le même plan : «Je perçois de plus en plus combien la musique est corrompue par les réticences et les conventions que lui impose le drame. Ainsi le public obtient de haute lutte un art de plus en plus démocratique, et à travers Wagner, il a affirmé dangereusement sa volonté de tyrannie (jusqu'où va ma méfiance ?). Cet hiver deux théâtres ont représenté ici Carmen, l'un en français, l'autre en italien et votre ami s'est obstinément interdit Carmen!). Retour de la musique, s'évadant de l'anti-naturel des acteurs, pour revenir à la nature même de la musique - qui en fin de compte, est la forme la plus idéale de la sincérité moderne ${ }^{3}$.

7 Pourquoi Nietzsche s'abstient-il même de Carmen? Pourquoi se défie-t-il de toute représentation musicale ? Parce que la musique n'a rien à gagner à être représenté. Toujours le drame prend le dessus sur la pure résonance, sur la musique absolue dont Liébert a raison de dire qu'au fond elle a été ce que Nietzsche a toujours recherché. Et n'hésitons pas maintenant à parler clairement: si la musique absolue est pour Nietzsche la seule musique possible, c'est parce que seule une musique débarrassée des multiples conventions humaines (y compris le langage et le mythe) nous rapporte à des rythmes que personne ne perçoit. Pour cela, il faut une troisième oreille, une oreille où l'éternelle araignée qu'on nomme raison n'a pas fait sa toile. Que ne doit-on à ce cérumen rationnel ? Que de surdités! Que de gravité ! «De la cire dans les oreilles, c'était là, jadis, presque la condition préalable au fait de philosopher: un authentique philosophe n'avait plus d'oreille pour la vie, pour autant que la vie est musique, il niait la musique de la vie »".

Wagner a-t-il composé avec de la cire dans les oreilles ? A-t-il été sourd à son tour à la musique de la vie? Difficile de le croire si l'on souvient quelque peu de l'enchantement que Wagner transmit à La Naissance de la tragédie: "Quand on a, comme ici, appliqué l'oreille aux pulsations de la volonté universelle, quand on a senti, dans toute sa fureur, le désir d'exister jaillir de ce cœur battant et se répandre tantôt avec le fracas du torrent, et tantôt un murmure de ruisseau, dans toutes les artères du monde - comment pourrait-on ne pas se briser d'un coup ? Enfermé dans la misérable coquille de verre, pourrait-on tolérer d'entendre l'écho de cette innombrable clameur de joie et de douleur qui monte de "l'immensité de la nuit des mondes ", sans qu'à l'appel de cette mélodie du berger on aille irrésistiblement chercher refuge dans sa patrie originelle?».

9 Que Nietzsche ait cru devoir atténuer cette expérience convulsive par la lumière apollinienne du mythe et du héros tragique, sous l'influence de Wagner lui-même, et qu'il ait cru que Wagner, par la suite, se mettait de plus en plus à distance de cette expérience première à force d'alourdir sa musique d'un élément dramatique, pire d'une dramaturgie mystico-chrétienne, cela peut-il le moins du monde atténuer cette première perception d'une incandescence radicale de la musique? Les pensées de Nietzsche sont moins sinueuses qu'on ne le dit. Après La Naissance de la tragédie, la question reste en vérité identique. Même interrogation en effet: à quelle sorte de musicalité la musique doit-elle nous ouvrir les oreilles ? À quel tumulte, à quel silence ? Certainement à autre chose qu'à l'affect intérieur, qu'à l'émoi sentimental. À une musique par-delà la musique même si ce n'est plus celle de l'être mais celle de la vie ; 
même si ce n'est plus celle de la transcendance des hauteurs mais celle de l'immanence de la terre ; même si ce n'est plus l'au-delà qui chante mais la volonté de puissance qui s'enroule dans un rythme stellaire. Bien sûr, Nietzsche a changé, et Wagner aussi. Wagner s'est enfermé selon Nietzsche dans la grande «imposture de la transcendance et de l'au-delà ». À force de tyranniser la musique par l'Idée, il est presque devenu le meilleur émule de Hegel. Rien de comparable ici à l'évolution nietzschéenne qui le porte vers la découverte du «rythme vivant» de l'univers ou Volonté de puissance. Transcendance contre immanence.

10 La solution diverge mais le problème est resté le même. Le jugement de Liébert à ce sujet nous paraît bien plus sûr que la littérature postmoderne empressé d'attribuer à Wagner toutes les fautes de l'histoire et à Nietzsche toutes les prophéties. «Adepte à peine déguisé de la musique absolue, Nietzsche au fond, restait imprégné de la métaphysique romantique. [...] En raison même de son absence d'objet et de destination précise, elle révélait l'infini : ce que Nietzsche appela d'abord le divin, puis des mondes supérieurs». Et faut-il ajouter à notre tour, la volonté de puissance sous la forme générique de la «vie " 5 .

11 Ainsi s'éclaire un texte, écrit en 1887, tout à fait capital pour comprendre ce qui s'est noué entre Nietzsche et Wagner : « Prélude de Parsifal, le plus grand bienfait qu'il m'ait été accordé depuis longtemps. La puissance et la rigueur du sentiment, indescriptible. Je ne connais rien qui saisisse le christianisme à une telle profondeur et qui porte si âprement à la compassion. Totalement sublime et ému - aucun peintre n'a su rendre comme Wagner une vision aussi indescriptiblement mélancolique et tendre. [...] Comme si après de nombreuses années quelqu'un me parlait enfin des problèmes qui m'inquiètent, non pas naturellement pour leur donner justement les solutions que je tiens prêtes, mais les réponses chrétiennes".

12 Sans crainte d'être entendu, Nietzsche se permet ici un aveu sincère et ému. Que seul, parmi les allemands, Wagner pensait dans le même horizon que lui. Ce qui signifie que les problèmes musicaux de Wagner étaient aussi les problèmes philosophiques de Nietzsche. Il suffit ici de refaire mentalement le chemin de pensée de Nietzsche depuis la première rencontre avec Wagner pour comprendre le sens des problèmes qui les unit: l'écho musical de l'infini, le sens du tragique, de la souffrance et de la grandeur de la souffrance, la passion du mystère, la nuit du monde qui est aussi minuit et lumière éternelle. Que Nietzsche ait donné sa réponse et que, par elle (la volonté de puissance comme principe cosmologique), il se soit détaché de Wagner n'enlève rien au fait que cette musique a ouvert sa pensée à ce que doit être la tâche de la pensée : consoner avec "la respiration du monde ", c'est-à-dire pour Nietzsche avec la puissance universelle, ondes et flux, rythmes et formes, pures intensités de devenir. Libérer la puissance la plus active.

13 Peu importe que telle ou telle musique soit plus en harmonie avec la vie et le principe immanent de la vie. Peu importe que Nietzsche se soit attaché, après tant d'autres musiciens, à Bizet. Cette préférence n'a de sens que sur fond d'une bien plus ample pensée, d'une expérience originaire indépassable, commune à Nietzsche et à Wagner, et que réalisent plus ou moins les autres musiques. Même le renoncement à Wagner prend l'allure d'un éloge de Wagner. Pourvu seulement qu'on tende l'oreille à la musique de la vie plutôt qu'aux tambours de la modernité, on percevra que cet éloge scintille encore dans textes les plus obscurs et anti-wagnériens de Nietzsche. 


\section{NOTES}

1.. Première variante du $\S 2$ de pourquoi j'écris de si bons livres de Ecce homo.

2.. Lettre à Peter Gast du 21 janvier 1887.

3.. Lettres à Peter Gast, Nice, premier avril 1887.

4.. Le Gai Savoir, § 372.

5.. LIÉBERT, Nietzsche et la musique, Paris, PUF, 1995, p. 223.

\section{RÉSUMÉS}

Pourquoi Nietzsche n'abandonna-t-il jamais Wagner même s'il se sépara de lui pour des motifs philosophiques? On sait que la musique de Wagner a joué le rôle de scène originaire dans la formation d'une pensée qui chercha initialement à comprendre le monde au travers d'une métaphysique esthétique. Il faut ajouter que la musique wagnérienne, en dépit d'une teneur esthétique et dramatique de plus en plus éloignée de ce qu'attendait Nietzsche, fut intégrée dans le centre vivant de la pensée nietzschéenne en tant qu'elle était la seule à poser musicalement les problèmes philosophiques de Nietzsche. Mes problèmes philosophiques, avouera tardivement Nietzsche, c'est cela que j'entends dans la musique de Wagner. 